\title{
Hoarseness as an Initial Symptom of Thymic Carcinoma: A Case Report
}

\author{
Shingo Takeuchi ${ }^{1}$, Naoyuki Yoshino ${ }^{1}$ and Jitsuo Usuda ${ }^{2}$ \\ ${ }^{1}$ Department of Thoracic Surgery, Nippon Medical School Tama Nagayama Hospital, Tokyo, Japan \\ ${ }^{2}$ Department of Thoracic Surgery, Nippon Medical School, Tokyo, Japan
}

\begin{abstract}
A 65-year-old man was referred to our hospital for evaluation of hoarseness and deteriorating voice. Computed tomography revealed an area of poor contrast enhancement in soft tissue (size, $30 \mathrm{~mm}$ ) in the inferior pole of the thyroid gland, extending to the upper margin of the sternum. No infiltration of blood vessels or bones, and no significant swelling of neck lymph nodes, was observed. These findings suggested a diagnosis of thymoma, thymic carcinoma, or thyroid tumor. Surgery was performed via median sternotomy, and complete thymectomy and tumor excision accompanied by partial thyroidectomy were performed. Histopathological examination revealed atypical polygonal tumor cells in a sheet-like arrangement, which formed a solid proliferative focus, and squamous cell carcinoma with infiltrative growth was diagnosed. Postoperatively, radiotherapy (60 Gy) was administered to the superior mediastinum. The patient is alive 22 months after surgery, without recurrence.
\end{abstract}

(J Nippon Med Sch 2019; 86: 357-359)

Key words: hoarseness, thymic carcinoma

\section{Introduction}

Thymic carcinoma is highly aggressive and has a poor prognosis. When thymic carcinoma is diagnosed on the basis of the presence of symptoms, radical surgery is a challenge because of the high probability that the tumor has already invaded surrounding organs. We report a case of thymic carcinoma diagnosed because of an initial symptom of hoarseness.

\section{Case Report}

A 65-year-old man with hoarseness and a deteriorating voice received an initial diagnosis of idiopathic recurrent nerve paralysis at an otorhinolaryngology clinic. The patient's condition did not improve with the use of steroids and other standard treatments, and he was referred to our hospital for detailed evaluation. Chest radiography showed no evident abnormalities. Computed tomography revealed an area of poor contrast enhancement (size, $30 \mathrm{~mm}$ ) in soft tissue in the inferior pole of the thyroid, extending to the upper margin of the sternum (Fig. 1). Magnetic resonance (MR) imaging revealed a tumorous lesion (maximum dimensions: $24 \mathrm{~mm} \times 34 \mathrm{~mm}$ ) anterior to the trachea and in contact with the inferior pole of the thyroid. Internally, the lesion had a signal intensity approximately equivalent to that of muscle on T1-weighted images and a slightly lower signal intensity on T2weighted images. The contrast-enhanced MR image showed uniform, weak contrast enhancement (Fig. 2). No infiltration of blood vessels or bones was evident, which suggested a diagnosis of thymoma, thymic carcinoma, or thyroid tumor. There was no significant swelling in neck lymph nodes.

Fluorodeoxyglucose positron emission tomography (FDG-PET) revealed moderate accumulation of glucose in the mass: the standardized uptake values were 4.03 and 3.93 in the early and late phases, respectively. A tumorous lesion was considered probable; however, determining whether it was benign or malignant in nature was difficult because of the moderate accumulation of glucose on FDG-PET. Additionally, acetylcholine receptor antibody levels were below the criterion level and thyroid function was normal. Thus, a tumor of thymic or thyroid origin was suspected, and surgery via median sternotomy was performed.

Correspondence to Shingo Takeuchi, MD, PhD, Department of Thoracic Surgery, Nippon Medical School Tama Nagayama Hospital, 1-7-1 Nagayama, Tama, Tokyo 206-8512, Japan

E-mail: take0818@nms.ac.jp

https://doi.org/10.1272/jnms.JNMS.2019_86-605

Journal Website (https://www.nms.ac.jp/sh/jnms/) 


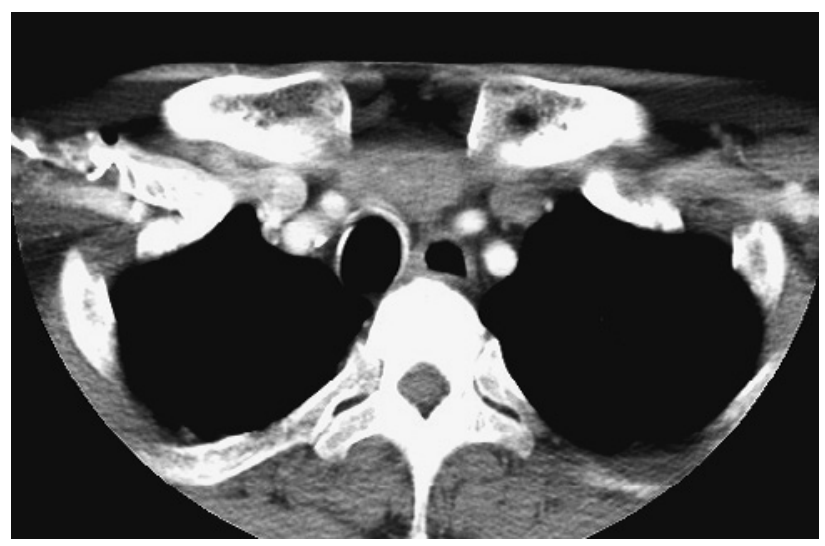

Fig. 1 A computed tomography scan showed an area of poor contrast enhancement of soft tissue $(30 \mathrm{~mm}$ in size) in the inferior pole of the thyroid, extending to the upper margin of the sternum.

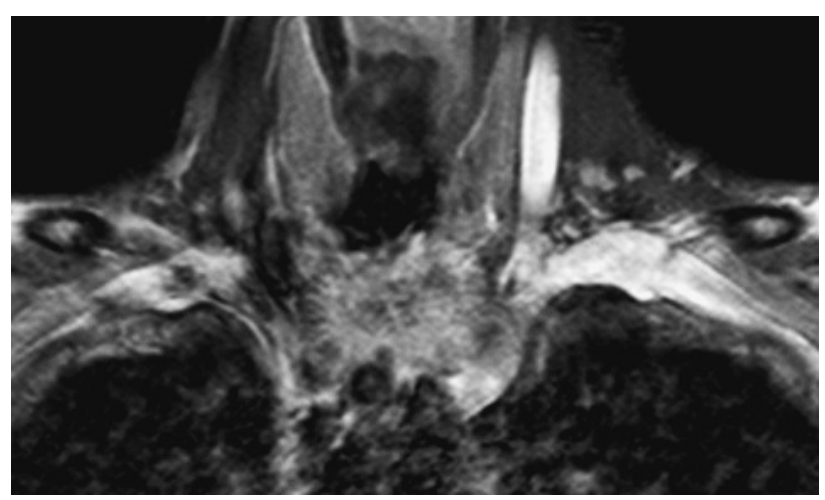

Fig. 2 Magnetic resonance (MR) imaging revealed a tumorous lesion (maximum dimensions: $24 \mathrm{~mm} \times 34$ $\mathrm{mm}$ ) anterior to the trachea and in contact with the inferior pole of the thyroid. Internally, the lesion had a signal intensity approximately equivalent to that of muscle on T1-weighted images and a slightly lower signal intensity on T2-weighted images.

The tumor, which was determined to have originated in the thymus, was located in the superior mediastinum, facing the anterior plane of the trachea. Infiltration of the inferior pole of the thyroid was suspected; therefore, complete thymectomy and tumor excision accompanied by partial thyroidectomy were performed. In addition, the left recurrent nerve was resected along with the tumor. Histopathological examination showed atypical polygonal tumor cells in a sheet-like arrangement, which formed a solid proliferative focus. Thus, squamous cell carcinoma with infiltrative growth was diagnosed (Fig. 3). Residual lymph tissue was present around the tumor, and small amounts of lymph tissue were present sporadically within fat tissue, suggesting a tumor of thymic origin. Furthermore, infiltration by squamous cell carcinoma

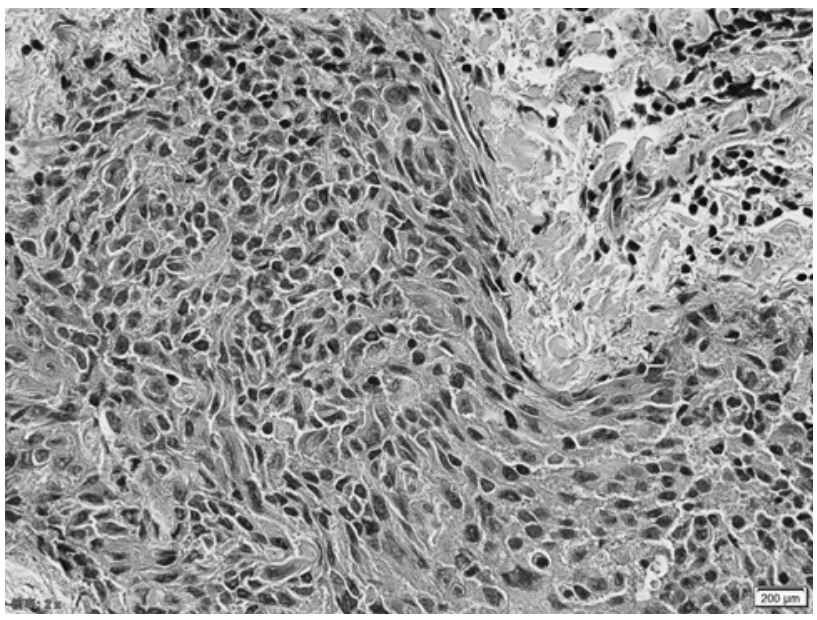

Fig. 3 Histopathological examination of the resected specimen showed atypical polygonal tumor cells in a sheet-like arrangement, forming a solid proliferative focus (hematoxylin-eosin staining).

was noted within the lymph and fat tissues. Tumor necrosis, cholesterin crystal precipitation, and replacement with fibrous scar tissue were observed. The tumor had invaded beyond the thymic capsule into the striated muscle and thyroid. Postoperatively, radiotherapy (60 Gy) was administered to the superior mediastinum. The patient is alive 22 months after surgery, without recurrence.

\section{Discussion and Conclusion}

Herein, we report a rare case of thymic carcinoma in a patient presenting with hoarseness who successfully underwent complete resection and achieved long-term survival.

Thymic epithelial tumors comprise thymomas, thymic carcinomas, and thymic carcinoid tumors. Thymic carcinoma is an uncommon neoplasm of the anterior mediastinum and has a poor prognosis. The tumor is highly aggressive, and local intra- and extrathoracic metastases occur in most patients ${ }^{1}$. In a review of 60 patients with thymic carcinoma, survival rates at 1, 3, and 5 years were $57 \%, 40 \%$, and $33 \%$, respectively ${ }^{2}$. Several case series reported that thymic carcinomas account for approximately $10 \%$ to $20 \%$ of all thymic epithelial tumors and that most patients are middle-aged adults ${ }^{3-6}$. In most case series, men and women were affected at the same rates, although several reports suggested that thymic carcinomas might be more prevalent in $\operatorname{men}^{4-12}$. The average age of patients with thymic carcinoma (46 years) does not significantly differ from that of patients with thymoma (48 years $)^{1,2}$. The most common histological type of thymic 
carcinoma is squamous cell carcinoma ${ }^{10-13}$. The dire prognosis for thymic carcinoma is worse than that for thymoma, and most affected patients exhibit locoregional invasion at the time of diagnosis ${ }^{5}$.

Compared with thymoma, thymic carcinoma is more likely to be invasive, as indicated by computed tomography scans, and most patients have clinical symptoms due to compression of mediastinal vital structures by the tumor $^{11}$. In a series of patients with thymic carcinoma, $68 \%$ presented with symptoms that were directly attributable to the mediastinal mass. Chest pain, cough, dyspnea, hoarseness, and dysphagia may occur because of compression of the trachea, recurrent laryngeal nerve, or esophagus ${ }^{2}$. When thymic carcinoma is diagnosed symptomatically, the tumor is likely to have invaded surrounding organs, making radical surgery a challenge. In the present case, hoarseness was the initial symptom. The deterioration of the patient's voice was attributable to compression and invasion of the recurrent nerve, and limited evidence suggests that such deterioration is a symptom of thymic carcinoma ${ }^{14}$. Thus, radical surgery is likely to be a challenge in patients presenting with hoarseness.

The gold standard treatment for resectable thymic carcinoma is surgery. Chemotherapy and radiotherapy are

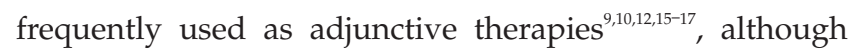
evidence for the utility of such therapies for thymic carcinoma treatment is equivocal. The optimal treatment for thymic carcinoma, with respect to overall survival, is complete resection ${ }^{10,16}$; chemotherapy is not the modality of choice for primary treatment of thymic carcinoma. In addition, several reports have concluded that radiotherapy is beneficial for local and disease-free survival of patients with thymoma and thymic carcinoma ${ }^{4,5,10,12,1516}$. Evidence from several studies ${ }^{3-6}$ suggests that postoperative radiotherapy is useful, even after complete resection.

In the present case, thymic carcinoma developed in the superior mediastinum, which is an uncommon location for this cancer type. Because of its nature, hoarseness (as a symptom of recurrent nerve paralysis) appeared before tumor invasion of vital organs, thus allowing relatively early tumor detection and complete resection. Hoarseness is not a common initial symptom of thymic carcinoma; however, in patients presenting with this symptom, the possibility of an anterior mediastinal tumor, such as thymic carcinoma, should be considered.

Conflict of Interest: The authors declare no conflicts of interest.

\section{References}

1. Quagliano PV: Thymic carcinoma: Case reports and review. J Thorac Imaging 1996; 11: 66-74.

2. Suster S, Rosai J: Thymic carcinoma. A clinicopathologic study of 60 cases. Cancer 1991; 67: 1025-1032.

3. Carillo C, Diso D, Mantovani S, Pecoraro Y, De Giacomo T, Ciccone AM, Poggi C, Longo F, Cassese R, Tombolini V, Rendina EA, Venuta F, Anile M: Multimodality treatment of stage II thymic tumours. J Thorac Dis 2017; 9: 2369-2374.

4. Ruffini E, Detterbeck F, Van Raemdonck D, Rocco G, Thomas P, Weder W, Brunelli A, Guerrera F, Keshavjee S, Altorki N, Schützner J, Arame A, Spaggiari L, Lim E, Toker A, Venuta F: European Society of Thoracic Surgeons Thymic Working Group. Thymic carcinoma: a cohort study of patients from the European society of thoracic surgeons database. J Thorac Oncol 2014; 9: 541-548.

5. Hamaji M, Shah RM, Ali SO, Bettenhausen A, Lee HS, Burt BM: A meta-analysis of postoperative radiotherapy for thymic carcinoma. Ann Thorac Surg 2017; 103: 16681675 .

6. Venuta F, Rendina EA, Anile M, de Giacomo T, Vitolo D, Coloni GF: Thymoma and thymic carcinoma. Gen Thorac Cardiovasc Surg 2012; 60: 1-12.

7. Kuo TT, Chang JP, Lin FJ, Wu WC, Chang CH: Thymic carcinomas: Histopathological varieties and immunohistochemical study. Am J Surg Pathol 1990; 14: 24-34.

8. Shimosato Y, Kameya T, Nagai K, Suemasu K: Squamous cell carcinoma of the thymus. An analysis of eight cases. Am J Surg Pathol 1977; 1: 109-121.

9. Marino M, Müller-Hermelink HK: Thymoma and thymic carcinoma. Relation of thymoma epithelial cells to the cortical and medullary differentiation of thymus. Virchows Arch A Pathol Anat Histopathol 1985; 407: 119149.

10. Hishida T, Nomura S, Yano M, Asamura H, Yamashita M, Ohde Y, Kondo K, Date H, Okumura M, Nagai K: Japanese Association for Research on the Thymus (JART). Long-term outcome and prognostic factors of surgically treated thymic carcinoma: results of 306 cases from a Japanese Nationwide Database Study. Eur J Cardiothorac Surg 2016; 49: 835-841.

11. Hsu CP, Chen CY, Chen CL, Lin CT, Hsu NY, Wang JH, Wang PY: Thymic carcinoma. Ten years' experience in twenty patients. J Thorac Cardiovasc Surg 1994; 107: 615620.

12. Zhai $Y$, Hui Z, Ji W, Wang $X$, Liang J, Mao $Y$, Luo $Y$, Zou S, Lv J, Zhou Z, Chen D, Zhang H, Xiao Z, Wang L, Feng Q: A single-center analysis of the treatment and prognosis of patients with thymic carcinoma. Ann Thorac Surg 2017; 104: 1718-1724.

13. Yen YT, Lai WW, Chang KW, Chang KC, Lee SC, Lin SH, $\mathrm{Wu} \mathrm{MH}$, Tseng YL: Factors predicting recurrence and postrecurrence survival in completely resected thymic carcinoma. Ann Thorac Surg 2014; 97: 1169-1175.

14. Ruffini E, Venuta F: Management of thymic tumors: a European perspective. J Thorac Dis 2014; 6 (Suppl 2): S228-237. doi: 10.3978/j.issn.2072-1439.2014.04.19.

15. Weksler B, Dhupar R, Parikh V, Nason KS, Pennathur A, Ferson PF: Thymic carcinoma: a multivariate analysis of factors predictive of survival in 290 patients. Ann Thorac Surg 2013; 95: 299-303.

16. Ahmad U, Yao X, Detterbeck F, Huang J, Antonicelli A, Filosso PL, Ruffini E, Travis W, Jones DR, Zhan Y, Lucchi M, Rimner A: Thymic carcinoma outcomes and prognosis: results of an international analysis. J Thorac Cardiovasc Surg 2015; 149: s95-100.

17. Kuriyama S, Ohashi R, Matsuda K, Sugano K, Muraki K, Minakata K, Ishikawa M, Kodama Y, Oh S, Suzuki K, Takahashi K: Twelve cases of advanced thymic carcinoma: A clinical review. Nihon Kokyuki Gakkai Zasshi 2010; 48: 604-608. 